\title{
Jan Assmann \\ Politisierung durch Polarisierung. Zur impliziten Axiomatik altägyptischer Politik
}

\section{Drei Formen des Impliziten}

Jedes soziale, das heißt auf gesellschaftliche Wirksamkeit abzielende Handeln impliziert einen Horizont gemeinsamer Verhaltens- und Gelingensregeln sowie Wert- und Sinnvorstellungen ${ }^{1}$. Das Modell hierfür ist das sprachliche Handeln. Ohne die gemeinsame Kenntnis einer Sprache wäre sprachliches Handeln erfolglos. Die Regeln dieser Sprache bleiben jedoch normalerweise implizit und werden nur fallweise, beim Spracherwerb oder im Falle eines Mißverständnisses, explizit thematisiert. Eine zusammenhängende Thematisierung sprachlicher Regeln, d.h. eine explizite Grammatik, ist eine späte Errungenschaft ${ }^{2}$. Das Nachdenken über die Sprache, das „Sprachdenken“, begleitet aber das Sprechen lange vor dem Auftreten expliziter Grammatiken. Nichts wäre absurder als die Vorstellung, Sprachen, denen die Sprachwissenschaft noch keine wissenschaftliche Beschreibung hat angedeihen lassen, besäßen keine Grammatik. Nicht anders steht es mit dem politischen Denken. Auch politisches Handeln setzt die Geltung einer Art von Grammatik, eines „Codes“ voraus (dessen Einheiten freilich von anderer Art sind als sprachliche Regeln und dessen Geltung meist auch mit anderen Mitteln durchgesetzt wird). Auch politisches Handeln ist vom Nachdenken über diesen Code begleitet, lange vor dem Auftreten expliziter politischer Diskurse. Dieses Nachdenken mündet normalerweise in die politische Praxis. Nur in Ausnahmefällen und Krisensituationen kommt es zur Entstehung expliziter Diskurse. Was das pharaonische Ägypten betrifft, sind wir weitestgehend auf die Erschließung „impliziter Politologie“ angewiesen, auf die „impliziten Axiome“ politischen Handelns ${ }^{3}$.

${ }^{1}$ Vgl. hierzu Jürgen Habermas, Theorie des kommunikativen Handelns, 2 Bde. (Frankfurt 1981).

${ }^{2}$ Vielleicht ist es nicht ganz zufällig, daß ihr Auftreten, in Griechenland wie in Indien, mit dem ersten Auftreten expliziter politischer Diskurse zusammenfällt.

${ }^{3}$ Vgl. Wolfgang Huber, Ernst Petzold, Theo Sundermeier (Hrsg.), Implizite Axiome. Tiefenstrukturen des Denkens und Handelns (München 1990). 


\subsection{Handeln}

Die politische Praxis der alten Ägypter ist eindrucksvoll genug. Das braucht hier nicht ausgeführt zu werden. Es genügt, an einige wohlbekannte Tatsachen zu erinnern. Der pharaonische Staat ist die erste bedeutende Staatsgründung der Menschheitsgeschichte 4 . Wenn die Griechen „keine Griechen vor sich hatten“, dann gilt entsprechendes in viel schärferem Sinne für die Ägypter. Denn die Griechen hatten zumindest eine zivilisierte Staatenwelt und reiche kulturelle Traditionen vor und neben sich, an die man - wie immer transformativ, innovativ und auch kontradistinktiv - anknüpfen konnte. Aber was lag dem ägyptischen Staat voraus? In wenigen Jahrhunderten, von circa 3100 bis 2700 v. Chr., vollzieht sich hier der Übergang von Clanverbänden und Häuptlingstümern zu einem einheitlichen und großräumigen, zentral organisierten, bürokratisch verwalteten Herrschaftsgebilde mit einheitlicher Sprache, Kultur, Religion, Kunst, Rechts- und Wirtschaftssystem, das allen späteren Staats- und Reichsgründungen als Vorbild dient ${ }^{5}$. Wenn die monotheistische Religion die spezifische Errungenschaft Israels darstellt, dann kann der monokratische Staat als die spezifische Errungenschaft Ägyptens gelten. Geschichtlich war sie nicht minder folgenschwer. Noch heute sind maßgebliche, vor allem konservative Staatstheorien entscheidend geprägt von Ideen, die in Ägypten erstmals greifbar werden.

Diese Greifbarkeit ist allerdings problematisch. Eine politische Praxis von der Größenordnung und geschichtlichen Strahlkraft der altägyptischen ist ohne ein begleitendes politisches Denken und ein konzeptionelles Arbeiten an den leitenden Ideen völlig unvorstellbar. Die Frage ist nur: wie kommen wir an diese konzeptionellen „Tiefenstrukturen“ des politischen Denkens und Handelns der alten Ägypter heran? Hierfür gibt es drei Wege. Der eine besteht in einer Analyse der politischen Praxis selbst, soweit sie uns durch die Quellen zugänglich wird. Hier geht es um eine Rekonstruktion des Handlungs- und Entscheidungsspielraums sowie der handlungsleitenden Axiome, die sich auf das reiche Korpus der Königsinschriften stützen kann, in denen politisches Handeln nicht nur beschrieben, sondern auch teilweise recht ausführlich begründet wird. Diesen Weg, dem ich vor Jahren eine eigene Studie gewid-

${ }^{4}$ Die Stadtstaaten des Vorderen Orients und der Indus-Kultur sind wesentlich kleiner. Großräumigere politische Gebilde entstehen hier „aszendent“, durch Verträge, Allianzen, Amphiktyonien. In Ägypten entsteht umgekehrt eine stadtzentrierte Regionalstruktur „deszendent“ durch einen Prozeß der inneren Kolonisation und administrativen Aufgliederung des politischen Großraums.

5 Vgl. einstweilen Hans-Jürgen Niedenzu, Die Entstehung von herrschaftlich organisierten Gesellschaften. Eine Auseinandersetzung mit den Evolutionskonzepten von Habermas und Eder sowie eine Darstellung der Entstehung der ägyptischen Hochkultur (Frankfurt 1982); Michael A. Hoffman, Egypt before the Pharaohs (London 1980); Michael Atzler, Untersuchungen zur Herausbildung von Herrschaftsformen in Ägypten (Hildesheim 1981); Erika Endesfelder, Beobachtungen zur Entstehung des ägyptischen Staates (unveröff. Habil. Schr. Berlin 1980). Der Komplex bedarf dringend einer gründlichen Aufarbeitung, die die Ergebnisse der neuesten Grabungen und der ethnoarchäologischen Komparatistik berücksichtigt. 
met habe ${ }^{6}$, will ich im folgenden nicht beschreiten. Statt dessen wollen wir auf zwei anderen Wegen eine Annäherung an die implizite Politologie der altägyptischen Kultur versuchen.

\subsection{Symbolik}

Kein Satz trifft besser das Grundprinzip der altägyptischen politischen Symbolik als die Regel des radikalen Konstruktivismus: „Draw a distinction and you create a universe“, oder: „wenn ein Raum geteilt wird, entsteht ein Universum“ “. Die politische Symbolik des alten Ägypten, die Formen, in denen die Ägypter ihren Staat konzeptualisierten, kreisen um die Motive von (Zwei-)Teilung und Vereinigung, Zweiheit und Einheit. Ägypten heißt „die beiden Länder“ oder „die beiden Ufer“. Gemeint ist einmal eine Zweiteilung in Nord-Süd-Richtung, das andere Mal eine in Ost-West-Richtung. Die „beiden Länder" sind Ober- und Unterägypten, ägyptisch Schema ${ }^{c}$ und Mehu, also zwei ganz verschiedene Wörter. Der ägyptische König trägt zwei Titel: njswt als König von Ober-, bjt als König von Unterägypten. Seine beiden Kronen symbolisieren die Herrschaft über die beiden Landesteile und sind zwei Kronengöttinnen und zwei Kronenstädten zugeordnet, Hauptstädten mythischer (und vielleicht auch historischer) Vorläuferstaaten, die zum pharaonischen Reich vereinigt wurden ${ }^{8}$. „Vereinigung “ ist das Stichwort, das den Sinn dieser ausgeprägt dualistischen Symbolik am genauesten trifft. Nicht das statische Motiv der Zweiheit, der Grenze und Unterscheidung ist entscheidend, sondern das dynamische Motiv der Trennung und Vereinigung. Das zentrale politische Symbol, das mit Vorliebe z. B. auf den Seiten königlicher Throne dargestellt wird, ist eine Szene der Vereinigung: Horus und Seth schlingen und verknoten die Wappenpflanzen von Ober- und Unterägypten um ein längliches Gebilde herum, das eine Hieroglyphe mit der Bedeutung zm3, vereinigen“ ist (Abb. 1, S. 27). Der Staat, den der König beherrscht, ist das Resultat einer Vereinigung, die in der mythischen Urzeit die beiden Götter vollbracht haben und die jeder König bei Herrschaftsantritt und in der Ausübung seiner Herrschaft neu vollbringt ${ }^{9}$. Der Mythos von Horus und Seth ist als narrative Ausformung der Zweiheitssymbolik der Gründungsmythos des ägyptischen Staats. Das antagonistische Brüderpaar steht aber für mehr als nur für die geographische Zweiteilung in Ober- und Unterägypten. Hier stoßen wir zum ersten Mal auf das Prinzip der Polarisierung. Horus verkörpert die Zivilisation, Seth die Wildnis, Horus das Recht, Seth die Gewalt, Horus die Ordnung, Seth die Unordnung ${ }^{10}$. Einheit kann nur durch

${ }^{6}$ Politik zwischen Ritual und Dogma. Spielräume politischen Handelns im pharaonischen Ägypten, in: Saeculum 35 (1984) 97-114 = Stein und Zeit. Mensch und Gesellschaft im Alten Ägypten (München 1991) 238-258.

7 George Spencer Brown, Laws of Form (Neudruck New York 1979) 3, zitiert nach Siegfried J. Schmidt, Weisheit, oder (...), in: Aleida Assmann (Hrsg.), Weisheit (München 1990) 559.

${ }^{8} \mathrm{Zu}$ dieser Symbolik s. Eberhard Otto, Die Lehre von den beiden Ländern Ägyptens in der ägyptischen Religionsgeschichte, in: Studia Aegyptiaca I = Analecta Orientalia 17 (1938) 10-35.

${ }^{9}$ Vgl. zu dieser Symbolik Henri Frankfort, Kingship and the Gods (Chicago 1948); Jobn Gwyn Griffiths, The Conflict of Horus and Seth (Liverpool 1960). S. a. die treffenden Bemerkungen von Barry Kemp, Ancient Egypt. Anatomy of a Civilization (London 1989) 27-29.

${ }^{10} \mathrm{Zu}$ Seth vgl. Herman te Velde, Seth, God of Confusion (Leiden 1967); Erik Hornung, Seth. 
Versöhnung dieser antagonistischen Prinzipien, Versöhnung aber nur durch Unterwerfung des einen unter das andere hergestellt werden. Das Recht, die Kultur, die Ordnung müssen kämpfen und siegen; sie setzen sich nicht von selbst durch. Sie setzen sich aber nicht verdrängend an die Stelle von Chaos, Unordnung, Wildheit und Gewalt, sondern bändigen sie. Der Mythos fundiert daher keinen Zustand, sondern ein unabschließbares Projekt: die Bändigung des Chaos und die Herstellung von Ordnung durch Vereinigung. Die Einheit ist immer problematisch, sie ist niemals gegeben, sondern immer aufgegeben. Die durch diese dem König aufgegebene Vereinigung erzielte Einheit ist mehr als die Summe ihrer Teile: sie ist allumfassend. Die Herrschaft über die zwei Länder bedeutet Herrschaft über das Ganze, äg. „Allherrschaft“ ( $\mathrm{nb}$ tm) oder „Einherrschaft“ ( $n b$ $w^{c}$ ). Die beiden Länder vereinigen sich zur Welt. Wenn ein Raum geteilt, und - so würden die Ägypter fortfahren - die Teile vereinigt werden, entsteht ein Universum. Die Herrschaft über das Ganze bedeutet Herrschaft über die vom Sonnengott geschaffene und dem König überantwortete Welt.

Pharao ist also ein Weltherrscher. Aber diese "Welt“ ist ein mythischer, kein politischer Begriff. Es handelt sich nicht um das, was später Oikumene, die bewohnte Erde, genannt werden wird. Er impliziert nicht das expansionistische Programm, erobernd bis ans Ende der Welt vorzudringen ${ }^{11}$. Die Grenzen der Welt liegen vor Augen: im Osten und Westen die Berge der Wüste, im Norden das Mittelländische Meer, im Süden der erste Katarakt. Die Grenzen umschreiben das Gebiet, in dem die „Menschen“ wohnen. Jenseits dieser Grenzen und nicht mehr in der geordneten Welt wohnen „die neun Bogen“, unzivilisierte Nomaden, die es nicht zu unterwerfen, sondern draußen zu halten gilt. Eine große Gruppe politischer Symbole, allen voran die bekannte Szene vom „Erschlagen der Feinde“, kreisen um die Idee der Abwehr, des Niederhaltens und Vertreibens (äg. d3r) (Abb. 2, S. 27). Wir können sie als „Außensymbolik“ zusammenfassen. Wichtiger aber und für das ägyptische Selbstverständnis entscheidender ist die Innensymbolik der Zweiheit und Vereinigung.

Der Sinn dieser Symbolik ist integrativ. Politische Symbolismen lassen sich nach dem Kriterium der dominanten Funktion einteilen in vorwiegend distinktive oder „limitische“ (E. W. Mühlmann) ${ }^{12}$ und vorwiegend integrative Symbole als Zeichen der Abgrenzung und als Zeichen der Einheit und Vereinigung. Natürlich haben alle abgrenzenden Symbole, z. B. die Beschneidung oder die Haltung des Sabbat im Juden-

\section{Fortsetzung Fußnote von Seite 15}

Geschichte und Bedeutung eines ägyptischen Gottes, in: Symbolon N.F. 2 (1975) 49-63; Hellmut Brunner, Seth und Apophis - Gegengötter im ägyptischen Pantheon?, in: Saeculum 34 (1983) 226-234.

11 Der ägyptische Expansionismus ist eine Erscheinung späterer Epochen, des Neuen Reichs (ab 1500 v.Chr.), s. dazu Paul Frandsen, Egyptian Imperialism, in: Mogens Trolle Larsen (Hrsg.), Power and Propaganda (Kopenhagen 1979) 167-90; Karola Zibelius-Chen, Die Ägyptische Expansion nach Nubien. Eine Darlegung der Grundfaktoren (Wiesbaden 1988); dies., Das Alte Ägypten, in: Iring Fetscher, Herfried Münkler (Hrsg.), Pipers Handbuch der politischen Ideen (München 1988) 113-134.

${ }_{12}$ Emil Wilhelm Müblmann, Ethnogonie und Ethnogenese. Theoretisch-ethnologische und ideologiekritische Studie, in: Studien zur Ethnogenese (Abh. d. Rheinisch-Westfälischen Akad. d. Wiss. 72, Opladen 1985) 9-28, bes. 18-22. 
tum, auch einen integrativen, Zusammengehörigkeit ausdrückenden Charakter und alle einheitstiftenden Sammlungszeichen auch einen distinktiven. Trotzdem läßt sich in den meisten Fällen so etwas wie eine dominante Funktion ausmachen.

Das Symbol der vereinigten Zweiheit ist ein ausgeprägt integratives Symbol. Der Mythos von Horus und Seth verwandelt die Erinnerung an eine ursprüngliche Zweioder Vielheit von Häuptlingstümern, die - nicht ohne Konflikt - zum pharaonischen Reich vereinigt wurden, in treibende, motivierende, richtungweisende Sinnenergie, in den Impuls zu immer erneuter Anstrebung und Herstellung von Einheit. Vielleicht liegt in solcher „Mythomotorik “13 das Geheimnis der einzigartigen Konstanz des ägyptischen Staates. Denn hier geht es ja nicht einfach um bloße Dauer, sondern vielmehr um die Kraft zu identischer Erneuerung, zur strukturellen Selbstreproduktion auch über schwere Zusammenbrüche hinweg.

Integration bedeutet, Einheiten als „Teile“ zu denken in bezug auf ein ihnen übergeordnetes Ganzes. Die typische Form, diese Beziehung im Symbol anschaulich zu machen, ist der Körper mit seinen Gliedern. In Ägypten steht von Anfang an die Körpersymbolik neben der Zweiheitssymbolik. Die einzelnen Gaue oder „Stätten“ Ägyptens bilden die Glieder eines Körpers, der mit dem Gott Atum (der Name bedeutet ägyptisch „das Ganze“, „das All“) identifiziert wird. In den Riten der sog. „Gliedervereinigung“ wird mit dem Körper des verstorbenen Herrschers zugleich auch die Einheit des mit seinem Tod zerfallenen Landes wiederhergestellt. In der Spätzeit gehen diese Riten mit der sich ausbreitenden Osiris-Religion eine neue Verbindung ein. Osiris, gewissermaßen die Personifikation des toten Herrschers, ist der zerstückelte Gott, dessen Körperteile auf die 42 Gaue des Landes verteilt wurden und dessen körperliche Restitution im Ritus zugleich die politische Einheit des Landes sicherstellt ${ }^{14}$. Es wäre $\mathrm{zu}$ untersuchen, ob von hier verborgene Verbindungslinien zur paulinischen Vorstellung der Ekklesia als Leib Christi laufen.

Das Zentralsymbol der pharaonischen Monokratie ist die Flügelsonne, die man gerne und nicht ganz zu Unrecht als das „Staatswappen“ Ägyptens bezeichnet (Abb. 3, S. 28). Mit ihren Flügeln symbolisiert sie das Weltumspannende der Herrschaft über „Alles, was die Sonne umkreist" ${ }^{45}$, und mit der Sonnenscheibe die Idee der Einheit: Eine Sonne, ein Herrscher ${ }^{16}$. Wie die Sonne die Götterwelt, so zentriert der König die Menschenwelt. Die Spannung von Pluralität und Zentralität findet sich auf allen Ebenen ägyptischen Denkens wieder. Was im Kosmos die Sonne und was in der Menschenwelt der König, leistet im Menschenbild das Herz als das zentrierende, lenkende Prinzip, das die Vielheit zur Einheit koordiniert ${ }^{17}$.

${ }^{13}$ Ich übernehme diesen Begriff (als Übersetzung von frz. mythomoteur) von Anthony D. Smith, The Ethnic Origins of Nations (Oxford 1986) passim, der ihn wiederum von J. Armstrong übernimmt.

${ }^{14}$ Vgl. Horst Beinlich, Die Osirisreliquien. Zum Motiv der Körperzergliederung in der altägyptischen Religion (Ägyptol. Abh. 42, Wiesbaden 1984).

${ }^{15}$ Entsprechende Wendungen sind seit dem Alten Reich belegt, werden aber wirklich häufig erst mit dem Neuen Reich.

${ }_{16}$ Zur Urbild-Abbild-Beziehung zwischen Sonne und Herrscher vgl. Anm. 18.

${ }_{17}$ Eine ganz andere Sicht vertritt Emma Brunner-Traut in: Frühformen des Erkennens (Darmstadt 1990), die den Aspekt der Vielheit verabsolutiert. 
Durch die Homologie zum monokratisch organisierten Kosmos gewinnt die Monokratie Pharaos den Charakter der Heiligkeit. Nur der Einherrscher setzt die Herrschaft des Einen Schöpfers fort, kann als Abbild der Sonne und Stellvertreter des Schöpfers gelten $^{18}$. Ihren im eigentlichen Sinne politischen Charakter gewinnt diese Idee der Einheit aber erst auf dem Hintergrund der Zweiheit, des Antagonismus und der Polarisierung. Erst als eine auf diesem Hintergrund ständig neu herzustellende und aufrechtzuerhaltende Einheit wird die Idee zum politischen Programm, zu einer Aufgabe, die unablässig eingeschärft und bewußt gehalten werden muß.

\subsection{Kompaktheit}

Die dritte Form von Implizitheit, in der uns das politische Denken der alten Ägypter entgegentritt, wird bedingt durch die Nichteigenständigkeit oder Nichtausdifferenziertheit des Politischen oder, positiv ausgedrückt, durch die „Kompaktheit“ der ägyptischen (wie überhaupt archaischen) Begriffsbildung. Die Begrifflichkeit, in der wir uns über diese Befunde verständigen - z. B. Recht, Moral, Weisheit, Religion, Politik, Herrschaft, Macht, Gewalt usw. -, ist das Ergebnis jahrtausendelanger Prozesse begrifflicher Differenzierung. Dem ägyptischen Denken liegen solche Unterscheidungen fern. Alle politischen und rechtlichen Begriffe gehören in ein Wortfeld der Verbindlichkeit, das vier Dimensionen ungeschieden in eins setzt: Moral, Recht, Politik und Religion. Man könnte sich auf den Standpunkt stellen, daß von Politik und politischem Denken überhaupt erst dort die Rede sein kann, wo sich dieser Bereich sozialen Handelns als autonome Sinndimension aus dem Gesamtkomplex ausdifferenziert und in entsprechenden Diskursen expliziert hat ${ }^{19}$. Dagegen ist nichts einzuwenden; nur gilt Entsprechendes dann ebenso für Moral, Recht und Religion. Hier hat sich aber ein Sprachgebrauch eingebürgert, der diese Begriffe ohne weiteres auch auf alle möglichen Befunde einfacher und früher Gesellschaften anwendet, deren Eigenbegrifflichkeit noch die kompakte Ungeschiedenheit des Verbindlichen widerspiegelt. Auch dieser Sprachgebrauch ist legitim, sofern er sich des Unterschieds bewußt bleibt und die entsprechenden Begriffe als solche nicht der Quellensprache, sondern der wissenschaftlichen Metasprache verwendet. In diesem Sinne möchte ich auch hier mit Bezug auf das alte Ägypten von politischem Denken sprechen und mich damit auf die begrifflich nicht ausdifferenzierte Sinndimension des Politischen beziehen, wie sie im

${ }^{18}$ In dieser Beziehung mutueller Repräsentation irdischer und göttlicher Herrschaft liegt das Grundproblem der „Politischen Theologie“, wie es Erik Peterson in seinem berühmten Traktat von 1935 entfaltet hat: Monotheismus als politisches Problem, nachgedruckt in Erik Peterson, Theologische Traktate (München 1951) 45-147. S. dazu Alfred Schindler (Hrsg.), Monotheismus als politisches Problem. Erik Peterson und die Kritik der politischen Theologie (Gütersloh 1978) sowie Alfred Schindler, Fritz Scholz, Die Theologie Carl Schmitts, in: Jacob Taubes (Hrsg.), Der Fürst dieser Welt (München 1983) 153-73. Für die (durchweg politische) Religion des Alten Ägypten ist diese Beziehung mutueller Repräsentation konstitutiv, aber sie hat nichts mit Monotheismus zu tun, sondern vielmehr mit dem, was Eric Voegelin „Summodeismus“ genannt hat, d.h. die Verehrung einer monokratisch zentrierten Götterwelt. Vgl. dazu Eric Voegelin, Order and History Bd. 1: Israel and Revelation (Louisiana 1956).

19 So etwa Christian Meier, Die Anfänge des politischen Denkens bei den Griechen (Frankfurt 1980). 
Begriffsfeld der Verbindlichkeit zusammen mit den Sinndimensionen des Moralischen, Rechtlichen und Religiösen mitkonnotiert wird.

Dafür bietet sich in erster Linie ein Begriff an, den wir im Deutschen mit „Wahrheit, Gerechtigkeit, Ordnung “ umschreiben: der Begriff $M a^{\prime} a t^{20}$. Wir fragen also nach dem politischen Denken der Ägypter, insoweit es in den Diskursen über die Gerechtigkeit als eine Sinndimension neben Moral, Recht und Religion miteingeschlossen ist.

\section{Konnektive Gerechtigkeit}

Es ist wohl nicht übertrieben, wenn man den Begriff der Gerechtigkeit, äg. Ma'at, als den Zentralbegriff der ägyptischen Kultur bezeichnet. Diese Aussage ist vielleicht überraschend, denn gewöhnlich verbindet man mit dem Begriff der Gerechtigkeit und seiner Vorrangstellung die Botschaft der israelitischen Propheten, die damit der Unmoral nicht nur ihrer eigenen Gesellschaft, sondern der gesamten „heidnischen "Welt entgegentreten. In Wirklichkeit geht es aber auch in der ägyptischen „Weisheit“ um nicht viel anderes als das, was die Propheten fordern ${ }^{21}$ : In der ägyptischen Weisheitsliteratur wird der Begriff der Gerechtigkeit zum Thema ausführlicher Erörterungen. Weisheitsliteratur ist Gerechtigkeitsliteratur. Der Begriff Ma'at, der ihr zentrales Thema bildet, hat hier genau den Platz inne, den in der biblischen Weisheitsliteratur der Begriff hokmab besetzt. Der Begriff der Gerechtigkeit bleibt also keineswegs implizit. Ganz im Gegenteil wird er diskurshaft entfaltet, und das im Rahmen einer Literatur, die das Zentrum der ägyptischen Kultur und ihrer normativen und formativen Ansprüche bildet. Wir fischen also keineswegs im Trüben, wenn wir nach dem impliziten politischen Denken der alten Ägypter fragen. Diese Frage trifft im Gegenteil auf einen reichen textlichen Befund, dann nämlich, wenn wir sie auf den „kompakten“ Begriff der Gerechtigkeit beziehen. Die Kompaktheit des ägyptischen Gerechtigkeitsbegriffs liegt, wie schon gesagt, in der Nichtunterscheidung seiner religiösen, moralischen, rechtlichen und politischen Sinndimension. Daher handelt es sich bei dieser Literatur um „ethico-theologico-politische Traktate“. Sie zerfallen in zwei Gattungen: Lebenslehren und Klagen. Die Lehren sind alle in die Form der väterlichen Unterweisung gekleidet und dienen der Einweisung der Söhne in die Tradition ${ }^{22}$. Interessanter für uns sind die Klagen. Ihr Adressat ist weniger festgelegt: der König, ein hoher Be-

${ }^{20}$ Vgl. hierzu Jan Assmann, Ma'at. Gerechtigkeit und Unsterblichkeit im alten Ägypten (München 1990). Eine kürzere französische Fassung erschien unter dem Titel Maât. L'Égypte pharaonique et l'idée de justice sociale (Paris 1989).

${ }^{21}$ Zur ägyptischen Weisheit s. die ausführlich eingeleitete und kommentierte Textsammlung von Hellmut Brunner, Ägyptische Weisheit. Lehren für das Leben (Zürich 1988). Ausgezeichnete englische Ubersetzungen der wichtigsten Texte finden sich auch bei Miriam Lichtheim, Ancient Egyptian Literature I: The Old and Middle Kingdoms (Berkeley-Los Angeles-London 1973).

${ }_{22}$ Vgl. hierzu auch Jan Assmann, Weisheit, Schrift und Literatur im alten Ägypten, in: Aleida Assmann (Hrsg.), Weisheit (München 1991) 475-500. Die Texte sind vollständig bei Brunner, a.a.O, übersetzt. 
amter, die eigene Seele, die Gesellschaft insgesamt, und entsprechend allgemeiner, grundsätzlicher und auch "politischer" ist ihr thematischer Skopus. Sie schildern eine Welt, die aus den Fugen geraten ist ${ }^{23}$ und machen dadurch klar, worin nach ihrer Vorstellung die „Verfugung“ der Welt besteht. Ihre Grundfrage ist: „was hält die Welt im Innersten zusammen?", und ihre Antwort: Gerechtigkeit. Daher bietet sich für diesen Gerechtigkeitsbegriff die Kennzeichnung als „konnektive Gerechtigkeit“, iustitia connectiva, $\mathrm{an}^{24}$.

Die konnektive Gerechtigkeit bindet den Menschen an den Mitmenschen und die Folge an die Tat. Sie stiftet einerseits Solidarität, andererseits den Tun-Ergehen-Zusammenhang. Sie wirkt daher verbindend nicht nur in der Sozial-, sondern auch in der Zeitdimension. Sie sorgt dafür, daß das Gute sich lohnt und das Böse sich rächt, sie organisiert die Zeit durch Erinnerung und Erwartung. Sie bewirkt, daß ein jeder seiner eingegangenen Verpflichtungen eingedenk ist und weiß, was er vom anderen zu erwarten hat. Sie ist mehr als ein "Code“ des „kommunikativen Handelns“, sie ist ein Vertrag. Ein Code ermöglicht, daß man einander versteht, ein Vertrag, daß man einander vertraut. Er hat einen stärker bindenden, verpflichtenden Charakter. Er wird niemals, wie ein Code, zur impliziten Selbstverständlichkeit, zur unreflektierten Gewohnheit, zum Habitus (im Sinne von P. Bourdieu) ${ }^{25}$, sondern muß immer neu bewußt gemacht und eingeschärft werden. Diese Reflexion und Einschärfung vollzieht sich im Diskurs der Weisheits- oder Gerechtigkeitsliteratur.

Man kann vier Typen konnektiver Gerechtigkeit unterscheiden, je nachdem, ob der moralische, der rechtliche, der religiöse oder der politische Aspekt dominiert.

(1) Wenn der moralische Aspekt im Vordergrund steht (das ist z. B. bei den Lebenslehren der Fall), geht es um „Gemeinsinn“, common sense, sensus communis. Das Gute lohnt sich, das Böse rächt sich, der sinnhafte Aufbau der (sozialen) Welt ist gewährleistet dann (und nur dann), wenn ein jeder lernt, sein individuelles Streben dem Gemeinwohl unterzuordnen, den Willen der Gemeinschaft zu seinem eigenen zu machen, sich dem Ganzen einzufügen, für seine Handlungen und deren Folgen ebenso einzustehen wie für seine Versprechungen und Gelöbnisse, Verantwortung zu übernehmen und dadurch Vertrauen zu erwerben und Vertrauen entgegenzubringen ${ }^{26}$. Solche Solidarität ermöglicht eine Ordnung, die den Beteiligten als selbstregulativ erscheint, als eine Art „immanenter Providenz ${ }^{“ 27}$, in der das Gute sich lohnt und das Böse sich rächt. Dann sieht es so aus, und wird gern so dargestellt, als walte zwischen Tat und Folge ein Automatismus oder eine Kausalität, die mit naturgesetzlicher Not-

\footnotetext{
${ }^{23}$ Vgl. Friedrich Junge, Die Welt der Klagen, in: Fragen an die altägyptische Literatur (Gedenkschrift E. Otto, Wiesbaden 1977) 275-284.

${ }^{24} \mathrm{Vgl}$. hierzu ausführlicher das dritte und neunte Kapitel meines Buches Ma'at. Gerechtigkeit und Unsterblichkeit.

${ }^{25}$ Pierre Bourdieu, Der Habitus als Vermittlung zwischen Struktur und Praxis, in: Zur Soziologie der symbolischen Formen (Frankfurt 1974) 125-158 (zuerst frz. 1970).

${ }^{26}$ Clifford Geertz, Common Sense as a Cultural System, in: Local Knowledge (New York 1983) 73-93.

${ }^{27}$ S. zu diesem Begriff Aleida Assmann, Was ist Weisheit? Wegmarken in einem weiten Feld, in: Weisheit (München 1990) 19.
} 
wendigkeit die Folge an die Tat bindet ${ }^{28}$. In Wirklichkeit handelt es sich aber um das Wirken der konnektiven Gerechtigkeit, die zu ihrem Funktionieren des Gemeinsinns, der gesellschaftlichen Solidarität, d.h. der Verpflichtung auf den zugrundeliegenden Vertrag bedarf.

(2) Wenn der rechtliche Aspekt im Vordergrund steht, wird das Funktionieren der konnektiven Gerechtigkeit in starken und dauerhaften Institutionen verankert, die das Böse mit Strafen belegen und dadurch dem Guten zum Durchbruch verhelfen. Die immanenten Regeln des harmonischen Zusammenlebens werden dann in Form eines Gesetzbuches kodifiziert. Dieser Aspekt der konnektiven Gerechtigkeit tritt besonders in Mesopotamien hervor.

(3) Wenn der religiöse Aspekt im Vordergrund steht, wird das Band zwischen Folge und Tat in die Hand der Götter oder Gottes gelegt. Jetzt ist es die Gottheit, die dafür sorgt, daß das Gute sich lohnt und das Böse sich rächt und die als Garant der Gerechtigkeit auftritt. Die Regeln des harmonischen Zusammenlebens werden zum göttlichen Willen erhoben, der vom Menschen entsprechendes Handeln fordert und den straft, der sich diesen Forderungen widersetzt. Dieser Aspekt tritt in Ägypten zumindest vor der Ramessidenzeit (13.-12. Jahrhundert) auffallend zurück; er ist besonders stark in Mesopotamien und natürlich in Israel ausgeprägt.

(4) Wenn der politische Aspekt im Vordergrund steht, wird das Funktionieren der konnektiven Gerechtigkeit dem Staat anheimgestellt. Jetzt sorgt der Staat für gesellschaftliche Solidarität und für den Tun-Ergehen-Zusammenhang. Die menschliche Gesellschaft ist zu schwach, die Götter zu weit entfernt oder zu ambivalent, um den Solidaritätsvertrag zu garantieren; dazu bedarf es vielmehr einer politischen, d.h. auf Herrschaft und Gehorsam basierenden Organisation. Das ist die ägyptische Auffassung, wie sie v.a. in den „Klagen“ entfaltet wird. Sie beschreiben das Chaos, das hereinbricht, wenn der Staat zerfällt. Wenn sich die herrschaftlich geformten Bindungen lockern, verschwinden Sinn und Ordnung aus der Welt. Das Gute lohnt sich nicht mehr, das Böse rächt sich nicht mehr, die Großen fressen die Kleinen und die Söhne erschlagen die Väter.

\section{Politisierung durch Polarisierung}

Polarisierung, die Spaltung der Welt in Freunde und Feinde, bedeutet Politisierung, d.h. Aufbau und Stärkung eines kollektiven Identitätsbewußtseins ${ }^{29}$. Soweit ist Carl Schmitts berühmt-berüchtigter Analyse unbedingt recht zu geben, auch wenn man nicht so weit gehen will, den Begriff des Politischen überhaupt in der Freund-Feind-

${ }^{28} \mathrm{Vgl}$. hierzu z. B. Klaus Koch, Gab es ein Vergeltungsdogma im Alten Testament?, in: Zeitschr. f. Theol. u. Kirche 52 (1955) 1-42, der den Begriff der „schicksalswirkenden Tatsphäre“ eingeführt hat.

${ }^{29}$ Zur theoretischen Grundlegung dieses Kapitels vgl. Aleida und Jan Assmann, Kultur und Konflikt. Aspekte einer Theorie des unkommunikativen Handelns, in: Jan Assmann, Dietrich Harth (Hrsg.), Kultur und Konflikt (Frankfurt 1990) 11-48. 
Unterscheidung aufgehen zu lassen ${ }^{30}$. Wem es gelingt, das Gefühl der Bedrohung durch einen mächtigen Feind zu verbreiten, der wird große Gefolgschaft finden. Die Gefahr muß aber nicht unbedingt immer von konkreten Feinden, d.h. von rivalisierenden Gruppen, ausgehen. Sie kann auch in allgemeineren Bildern von Chaos und Umsturz festgemacht werden. Auch hier zeigt sich, ähnlich wie im Fall der „integrativen“ bzw. „distinktiven“ Symbolik, daß Ägypten den Antityp zu Israel vertritt. Israel bezieht sein Identitätsbewußtsein aus der Frontstellung gegenüber einem Ägypten, das Symbol ist für jede Form von Tyrannei, Unterdrückung und Heidentum ${ }^{31}$. Hier steht nicht Kultur gegen Chaos, sondern die eine gegen die andere Kultur. Ägypten geht den umgekehrten Weg.

\subsection{Negative Soziologie}

Die Ägypter gehen davon aus, daß die menschliche Gesellschaft von sich aus zur Ungleichheit tendiert, d.h. in Arme und Reiche, Schwache und Starke, Unterdrückte und Unterdrücker zerfällt. Aus dieser Einsicht lassen sich zwei verschiedene Folgerungen ableiten. Die eine nimmt die Ungleichheit als Ausdruck gottgewollter Ordnung und gelangt zur Idee einer kosmisch verankerten Klassen- oder gar Kastengliederung der Gesellschaft. Die andere nimmt die Ungleichheit im Gegenteil als Ausdruck von Unordnung, die der Mensch zwar nicht aufheben kann, der es aber doch mit allen Kräften gegenzusteuern gilt. Letzteres ist die ägyptische Position. Die Ungleichheit wird als Isfet, als Unrecht, Lüge und Unordnung, eingestuft und der Ma'at, der Gerechtigkeit, Wahrheit und Ordnung gegenübergestellt ${ }^{32}$.

Ma'at, das Gegenteil dieser natürlichen Ungleichheit, die sich mit Notwendigkeit einstellt, wenn man den Dingen ihren Lauf (d.h. den Menschen ihren Willen) läßt, ist nun aber nicht etwa Gleichheit; diese gilt offenbar als völlig außerhalb irdischer Realisierbarkeit. Das Gegenteil ist vielmehr eine Ordnung, ein „Solidaritätsvertrag“, in dessen Geltungsrahmen die Reichen für die Armen sorgen, die Starken die Schwachen nicht unterdrücken können, den sprichwörtlichen Witwen und Waisen zu ihrem Recht verholfen wird ${ }^{33}$. Ich-nenne dieses Prinzip „vertikale Solidarität“. Die Bindungen der Mitmenschlichkeit, um die es hier geht, wirken vor allem nach oben und unten. Sie binden den einzelnen ein in ein vertikales System des Austauschs von Schutz (nach unten) gegen Gehorsam und Loyalität (nach oben). Das Paradigma, dessen sich der Ägypter vornehmlich bedient, um diese Bindungen und diesen Austausch anschaulich zu machen, ist die Sprache, näherhin das Hören. Worauf es ankommt, ist eine Kunst des rechten Hörens, die sich von oben nach unten als gutes Zuhören, von unten nach oben dagegen als Beherzigen und Gehorchen realisiert. Je höher einer

${ }^{30}$ Vgl. hierzu Dietrich Conrad, Der Begriff des Politischen, die Gewalt und Gandhis gewaltlose politische Aktion, in: Kultur und Konflikt, 72-112; Heinrich Meier, Carl Schmitt, Leo Strauss und „Der Begriff des Politischen“. Zu einem Dialog unter Abwesenden (Stuttgart 1988).

${ }_{31}^{31}$ Vgl. hierzu Michael Walzer, Exodus and Revolution (New York 1985, dt. Berlin 1988).

32 Für die Einzelheiten s. Assmann, Ma'at.

${ }_{3}$ Vgl. hierzu die in meinem Buch Ma'at herangezogene Literatur. Dazu: H. K. Havice, The Concern for the Widow and the Fatherless in the Ancient Near East. A Case Study in O. T. Ethics (Ph. D. thesis, Yale University 1978). 
steht, desto größer ist seine Schutzverpflichtung nach unten, je tiefer einer steht, desto größer seine Loyalitätsverpflichtung nach oben. Daraus ergibt sich, daß das Königtum als die am höchsten stehende Instanz in allererster Linie eine Schutzinstitution ist. Ihre vornehmste Aufgabe ist es, wie es in der Lehre für Merikare heißt, „den Rücken des Schwachen zu stärken“:

Für sie schuf er (der Schöpfergott) Herrscher, im Ei‘ und Befehlshaber, um den Rücken des Armen zu stärken ${ }^{34}$.

Ganz oben in dieser Pyramide vertikaler Solidarität steht der Schöpfergott selbst, dem auch der König zu Gehorsam verpflichtet ist. Von Gott heißt es, daß er

das Flehen erhört dessen, der in Bedrängnis ist und sein Herz dem zuneigt, der zu ihm ruft, den Furchtsamen errettet aus der Hand des Gewalttätigen und Recht spricht zwischen dem Armen und dem Reichen ${ }^{35}$.

Aber er hat diese Aufgabe an den König delegiert. Er hat, wie es in einem anderen Text hëißt,

den König eingesetzt auf der Erde der Lebenden

für immer und ewig,

den Menschen Recht zu sprechen, die Götter zu besänftigen,

die Ma'at zu verwirklichen, die Isfet zu vernichten ${ }^{36}$.

Die Einsetzung des Königtums ist die Form, in der Gott sich um die Gerechtigkeit unter den Menschen kümmert; für alles weitere sind sie selbst verantwortlich. In diesem Punkt allerdings erfährt das ägyptische Weltbild gegen Ende des 2. Jahrtausends gewaltige Erschütterungen und Veränderungen, auf die einzugehen hier den Rahmen sprengen würde ${ }^{37}$.

\subsection{Negative Anthropologie}

Der Grundsatz der „negativen Soziologie“ besagt, daß die Menschen von sich aus zur Gemeinschaft unfähig sind. Sich selbst überlassen, tendieren sie zum Chaos, das heißt zu einem Zustand der Ungleichheit, in dem die Starken die Schwachen umbringen oder unterdrücken. In der altindischen Lehre, die auch als negative Soziologie einzustufen ist, heißt dieser Zustand „das Gesetz der Fische“: die Großen fressen die Kleinen $^{38}$. Hierhin gehören auch die ägyptischen Anschauungen über die Ungleichheit unter den Menschen. Aber damit ist zunächst noch keine moralische Unterscheidung impliziert. Sie ergibt sich erst aus einem moralischen Urteil über die Natur des Men-

${ }^{34}$ Lehre für König Merikare P 135 f., engl. Übers. Lichtbeim, Literature, 106.

35 Papyrus Kairo CG 58038, vgl. Jan Assmann, Re und Amun. Die Krise des polytheistischen Weltbilds im Ägypten der 18.-20. Dyn. (OBO 51, 1983) 176f.; Ma'at, 235.

${ }^{36} \mathrm{Zu}$ diesem Text s. Ma'at, 201-212.

37 Vgl. hierzu Jan Assmann, State and Religion in the New Kingdom, in: William Kelly Simpson (Hrsg.), Religion and Philosophy in Ancient Egypt (Yale Egyptological Studies 3, 1989) 55-89; Ma'at, 7. und 8. Kapitel.

${ }^{38}$ Louis Dumont, Homo Hierarchicus (Paris 1966), dt. Die Soziologie des Kastenwesens (Wien 1976) 351. 
schen, die man als „negative Anthropologie“ bezeichnen kann. Unter dem Begriff einer „negativen Anthropologie“ fassen wir alle Lehren zusammen, die den Menschen als „böse“ voraussetzen. Das prominenteste Beispiel einer solchen negativen Anthropologie ist die christliche Lehre von der Erbsünde, auf deren politische Implikationen unlängst E. Pagels aufmerksam gemacht hat ${ }^{39}$. Von einer solchen Auffassung sind die Ägypter weit entfernt. Ihre negative Anthropologie äußert sich darin, daß sie die Unterscheidung zwischen dem Starken und dem Schwachen moralisch interpretieren und den Starken mit dem Bösen gleichsetzen. Sie gehen davon aus, daß von Natur aus der Starke der Böse ist, daß also der seinem eigenen Willen anheimgegebene, „naturbelassene" Mensch die Chancen ausnutzt, die sich ihm bieten, seinen Vorteil auf Kosten anderer durchzusetzen. Nietzsche hat diese Gleichsetzung von „stark“ und „böse“, „schwach“ und „gut" bekanntlich als eine speziell jüdisch-christliche Errungenschaft angesehen und als "Ressentiment" und "Sklavenmoral“ gedeutet ${ }^{40}$. Max Weber ist ihm darin mit großer Zustimmung gefolgt ${ }^{41}$. Damals waren die ägyptischen und vorderorientalischen Quellen noch unzureichend erschlossen. Inzwischen ist deutlich geworden, daß es sich hier um ein gesamtorientalisches Gerechtigkeitsdenken handelt, an dem Israel lediglich auf seine besondere Weise Anteil hat.

Die „böse“ Natur des Menschen äußerst sich darin, daß er zu der Geselligkeit, auf die hin er doch angelegt ist, nicht in der Lage ist. Die Ägypter lokalisieren diese Unfähigkeit zur Sozialität in seinem Willen, ägyptisch „Herzen“. Der Schöpfer, so heißt es in einem Text, hat

die Menschen einen jeden gleich seinem Nächsten geschaffen und verboten, daß sie Isfet tun sollten.

Aber ihre Herzen haben (sein) Verbot übertreten.

Aus dieser Schwäche der menschlichen Natur leiten die Ägypter, genau wie die Inder, die Notwendigkeit des Staates ab. Das Chaos, vor dem der Staat die Menschen schützen soll, ist ein Chaos „von innen“, denn es kommt von der Gier, der Habsucht und Eigensucht, die nach ägyptischer Auffassung dem Herzen von Natur aus innewohnt und die durch Erziehung gebändigt und in Gemeinsinn umgeformt werden muß ${ }^{42}$. Die Menschen brauchen den Staat, der sie voreinander schützt und sie zur Mitmenschlichkeit erzieht. Hier zeigt sich der Zusammenhang zwischen der Anthropologie der Ägypter und ihrer erstaunlichsten Leistung: dem Staat. Wenn Carl Schmitt recht hat mit seiner These, daß „echte politische Theorien den Menschen als böse voraussetzen " ${ }^{43}$, dann handelt es sich auch bei den entsprechenden ägyptischen Anschauungen um „echte politische Theorien“. Wenigstens zeigt sich doch im Licht dieser

39 Elaine Pagels, Adam, Eve and the Serpent (New York 1988), bes. Kap. V, „The Politics of Paradise".

${ }^{40}$ Friedrich Nietzsche, Zur Genealogie der Moral, Erste Abhandlung: „gut und böse“, „gut und schlecht“", in: Werke in drei Bänden, hg. v. Karl Schlechta, II (München 1955).

41 E. Fleischmann, Max Weber, die Juden und das Ressentiment, in: Wolfgang Scblucbter (Hrsg.), Max Webers Studie über das antike Judentum (Frankfurt 1981) 263-86.

42 Wie sehr diese archaischen Ängste im Denken Carl Schmitts wiederkehren, zeigt z. B. die brillante Studie von Nicolaus Sombart, Die Angst vor dem Chaos. Zum Carl-Schmitt-Syndrom, in: Merkur 498 (Jg. 44 Heft 8 August 1990) 638-651.

${ }^{43}$ Der Begriff des Politischen (Berlin $\left.{ }^{2} 1932\right) 59 \mathrm{ff}$. 
überspitzten These, daß die ägyptischen Unterscheidungen zwischen Ma'at und Isfet, Ordnung und Chaos, Recht und Unrecht, Gut und Böse nicht nur einen moralischen, sondern auch einen politischen Sinn haben. Durch diese Polarisierung wird die Welt politisiert, d.h. so konstruiert, daß der Staat als unausweichliche Grundbedingung menschlichen Zusammenlebens erscheint.

Politisierung ist jedoch nicht Sache eines Schrittes, einer evolutionären Errungenschaft, die einmal vollzogen wird und dann selbstverständliche Gültigkeit behält. Politisierung ist vielmehr Sache eines Bewußtseins, das eingeschärft und ständig wachgehalten werden muß. Das geschieht typischerweise durch Erzeugung eines Bedrohungsbewußtseins ${ }^{44}$. Es wird nicht nur eine Grenze gezogen, es wird vor allem das Ausgegrenzte als Quelle ständiger Gefahr dargestellt. Der ägyptische Staat gründet sich auf ein Bedrohungsbewußtsein, das den Feind nicht in äußeren Stämmen, Völkern oder Staaten festmacht, sondern als das Prinzip einer Gegenkraft zur Gerechtigkeit identifiziert, die sowohl in der Natur des Menschen, wie der Gesellschaft, wie sogar in der des Kosmos überhaupt liegt. Diese Gegenkraft wird in Gestalt einer riesigen Wasserschlange namens Apopis verkörpert, von der man annimmt, daß sie der Sonnenbarke im Himmel und in der Unterwelt entgegentritt und sie mit Stillstand bedroht. Diese Schlange muß auch mit den Mitteln des Kults bekämpft werden. Dadurch wird der Sonnenlauf und mit diesem zugleich auch die staatliche und politische Ordnung auf Erden in Gang gehalten:
Wenn man die Osiris-Zeremonien vernachlässigt zu ihrer Zeit an diesem Ort ..
dann wird das Land seiner Gesetze beraubt sein und der Pöbel wird seine Oberen im Stich lassen und es gibt keine Befehle für die Menge.
Wenn man den Feind nicht köpft, den man vor sich hat aus Wachs, auf Papyrus oder aus Holz nach den Vorschriften des Rituals, dann werden sich die Fremdländer gegen Ägypten empören und Bürgerkrieg und Revolution im ganzen Land entstehen.
Man wird auf den König in seinem Palast nicht hören und das Land wird seiner Schutzwehr beraubt sein ${ }^{45}$.

Damit kommen wir auf das Phänomen der „Kompaktheit“ zurück. Es basiert auf dem Prinzip einer analogischen Imagination, die alles zueinander in Beziehung setzt: Kosmos und Gesellschaft, Kult und Politik, Magie und Herrschaft. Die Liturgie dieses Abwehrrituals gegen Apopis ist uns erhalten: sie basiert durchgängig auf dem Parallelismus zwischen kosmischen und politischen Feinden.

Das „Politische“ ist also in Ägypten nicht nur nicht ausdifferenziert als eigenständiger Bereich menschlichen Denkens und Handelns, es ist im Gegenteil explizit in eins gesetzt mit kultischen, kosmischen und individuellen Heilsvorstellungen. Diese Nichtunterscheidung wird man aber wohl nicht als „Fehlen“, sondern als eine besondere Form politischen Denkens einstufen müssen. Nach dieser Auffassung gibt es nur eine einzige Ordnung; sie erweist sich im Gelingen, und es kommt darauf an, sie in

\footnotetext{
44 vgl. A. u. J. Assmann, Kultur und Konflikt, 17 ff. §4: „Die Krise in den Köpfen: Über die Kultivierung von Bedrohungsbewußtsein".

45 Papyrus Jumilhac ed. Vandier, 129 f.; s. Assmann, Ma'at, $185 \mathrm{f}$.
} 
allen Sphären zugleich, in der kosmischen, kultischen, politischen und häuslichen Sphäre, zur Geltung zu bringen. Ein größerer Gegensatz zu dem, was sich in Griechenland und Israel an politischem Denken entwickelt, ist schwer vorstellbar. Interessant ist aber, daß in Ägypten selbst, und viel früher als in Israel und Griechenland, eine Gegenbewegung aufgetreten ist, die sowohl das analogische Denken als auch das Prinzip einer Politisierung durch Polarisierung in Bausch und Bogen verworfen hat. Ich meine die revolutionären Reformen König Echnatons (14. Jahrhundert v. Chr.). Darauf kann ich hier nicht näher eingehen, aber einige zentrale Punkte seien wenigstens angedeutet ${ }^{46}$.

1. Die Welt wird ent-polarisiert. In Amarna gibt es den kosmischen Feind nicht mehr, die Unterscheidung von gut und böse spielt keine Rolle.

2. Die Welt ist nicht bedroht und bedarf keiner Inganghaltung. Bildzauber, sakramentale Magie und analogische Imagination werden verworfen.

3. Die Welt wird entpolitisiert. Die Unterscheidung zwischen Freund und Feind, Ägypten und Fremdländern, Bündnispartnern und Bündnisgegnern verliert ihren Sinn. Die Sonne scheint über allen Völkern gleicherweise. Die diplomatische Korrespondenz spiegelt das politische Desinteresse Echnatons vollkommen wider ${ }^{47}$.

4. Die Welt wird entmoralisiert. Die Sonne läßt zwar durch ihr Licht alles Leben entstehen und in der durch ihre Bewegung hervorgebrachten Zeit sich entfalten, aber sie kümmert sich nicht um die Weinenden und schützt nicht die Schwachen gegen die Starken.

5. Die Herrschaft wird naturalisiert. An die Stelle des moralischen und politischen Prinzips des Staats tritt das vitalistische Prinzip der Natur. Der Mensch wird von einem politisch-moralischen Wesen zu einem natürlichen Wesen herabgestuft und mit allem, was in und von der Sonne lebt, auf eine Stufe gestellt. Die Abhängigkeit alles Lebendigen vom Licht wird als Herrschaft ausgelegt, die die Sonne über ihre Geschöpfe ausübt. Der König versteht sich nur als eine Art Korregent oder „Juniorpartner" in dieser natürlichen Herrschaft des Sonnengottes.

Dieses utopische Projekt einer Entpolitisierung der Gesellschaft hatte für ganze 20 Jahre Bestand, dann wurde es aufgegeben, verfolgt und vergessen. Die forcierte Außenpolitik der folgenden Jahrzehnte, sowohl auf militärischem wie auf diplomatischem Gebiet, zeigt, in wie starkem Maße es hier bei dieser religiösen Umsturzbewegung auch um politische Entscheidungen ging.

${ }^{46}$ Für eine ausführlichere Darstellung dieser Deutung der Amarna-Religion s. Ma'at, 231-36.

47 William L. Moran, Les lettres d'El Amarna. Correspondance diplomatique du Pharaon (Paris 1987). 

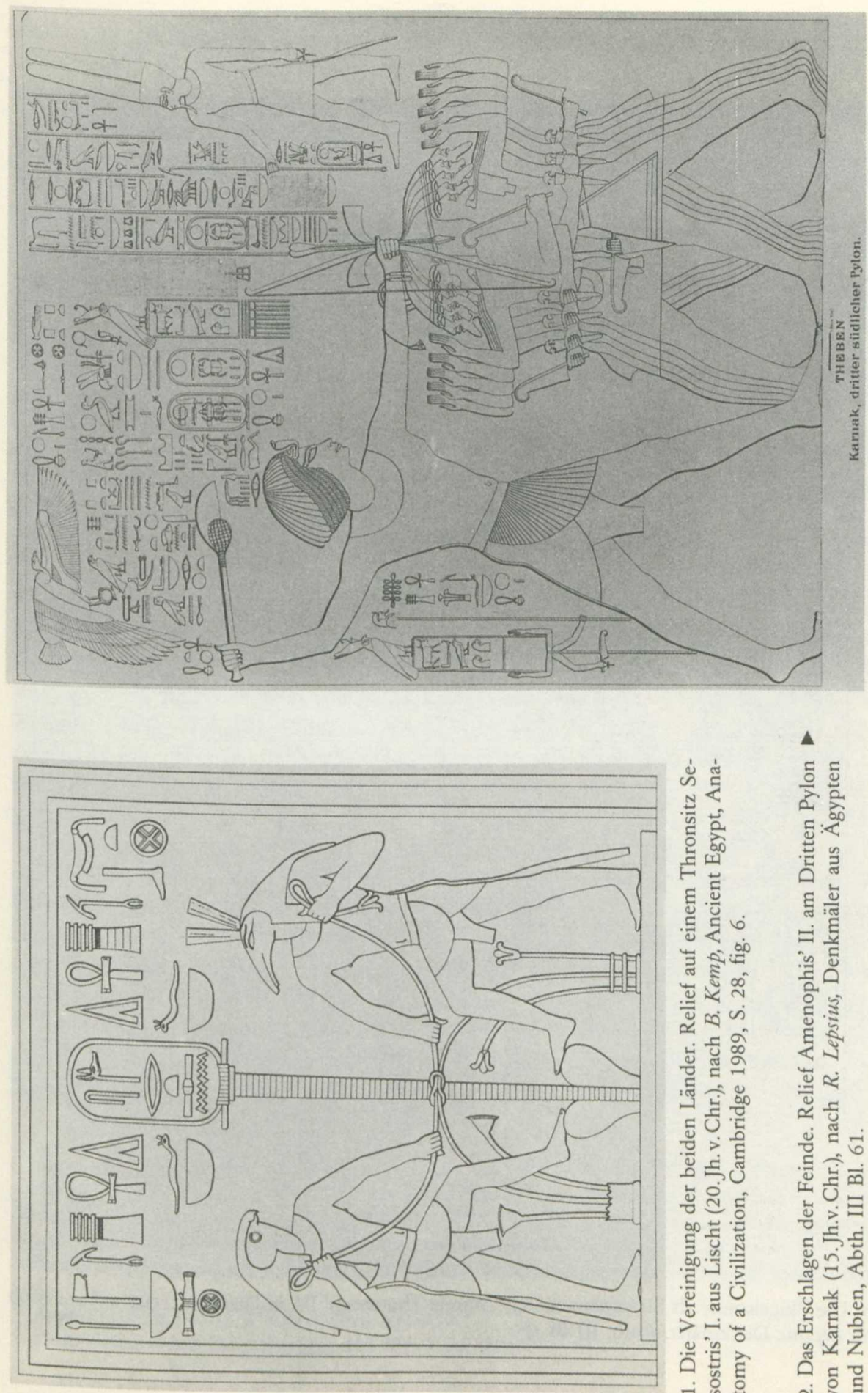

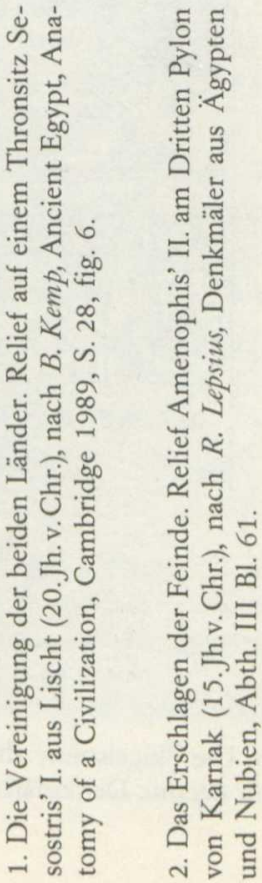




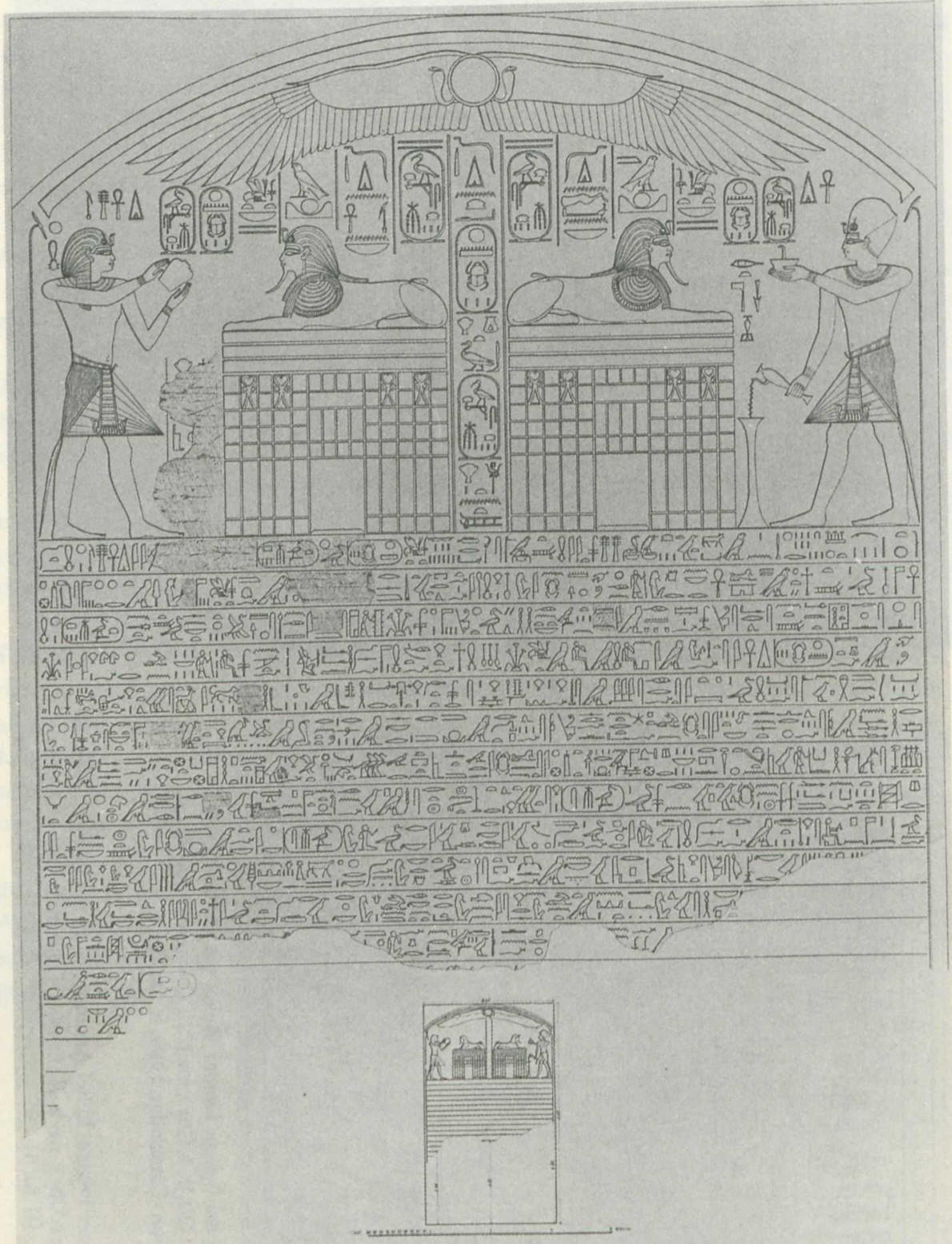

Pyramiden von Giseh.

Stele vor dem grofsen Sphinx.

3. Die Flügelsonne als Staatswappen. Sphinxstele Thutmosis' IV. in Giza (um 1400 v. Chr.), nach R. Lepsius, Denkmäler, Abth. III Bl. 68. 